\title{
Two-dimensional refractive index profiling by using differential near-field scanning optical microscopy
}

\author{
Wan-Shao Tsai and Way-Seen Wang \\ Graduate Institute of Electronics Engineering, National Taiwan University, No. 1, Section 4, Roosevelt \\ Road, Taipei, Taiwan 10617, Republic of China \\ Pei-Kuen Wei ${ }^{a}$ \\ Research Center for Applied Sciences, Academia Sinica, No. 128, Section 2, Academic Road, Taipei, Taiwan \\ 11529, Republic of China
}

(Received 6 June 2007; accepted 17 July 2007; published online 10 August 2007)

\begin{abstract}
The authors present two-dimensional reconstruction of the refractive index profile of an optical waveguide by using differential near-field optical microscopy. Using an inverse algorithm, the refractive index distribution is obtained directly from the measured optical near-field and its derivatives without any assumption of the index profile. The proposed method also takes advantage of subwavelength resolution and low noises in the waveguide region. Two-dimensional index profile reconstruction of a single mode fiber is measured for the demonstration. The measured optical field distribution and refractive index profile agree quite well with the calculated mode and the known index profile. (C) 2007 American Institute of Physics. [DOI: 10.1063/1.2769396]
\end{abstract}

Refractive index profile of an optical waveguide is crucial in the determination of waveguide mode properties. In principle, refractive index profile can be reconstructed by an inverse calculation algorithm ${ }^{1,2}$ when optical intensity distribution and its first and second derivatives are known. Conventional method that uses an objective lens and a chargecoupled device camera ${ }^{1-4}$ can only take the guiding mode image with micrometer spatial resolution. The impulsive and high frequency noise in the measured intensity profile make it almost impossible to take the differentiations. Several attempts have been made to overcome this problem by spatial smoothing of the measured intensity. For one-dimensional index profile, spatial smoothing is possible by fitting a curve using local least-squares method. For two-dimensional index profiles, the measured optical profile is usually smoothed by fitting with an analytical function or using a low-pass filter. It imposes constraints on the profile to follow the function and causes errors in the refractive index reconstruction. In this work, we propose a method to solve the differentiation problem by using a differential near-field scanning optical microscopy (DNSOM). With the proposed DNSOM system, not only the guiding mode distribution but also its first and second derivatives are measured simultaneously. It directly reconstructs the index profile with the inverse algorithm. No fitting functions and filters are used in the calculations. Moreover, with a tapered fiber probe, subwavelength optical resolution is achieved. ${ }^{5-7}$ A single mode fiber operated at $632 \mathrm{~nm}$ is measured for a demonstration of the reconstruction of two-dimensional index profile. The measured optical field distribution and refractive index profile agree quite well with the calculated mode and the known index profile.

The algorithm for solving the refractive index profile $n(x)$ from the measured intensity distribution $I(x)$ is based on the scalar wave equation. For most optical waveguide, the change of refractive index $\Delta n(x, y)$ is very small; it can be approximated by a combination of the substrate index $n_{s}$ and

\footnotetext{
a) Author to whom correspondence should be addressed; electronic mail: pkwei@gate.sinica.edu.tw
}

$\Delta n(x, y), \quad$ as given by $n^{2}(x)=\left[n_{s}+\Delta n(x, y)\right]^{2} \approx n_{s}^{2}$ $+2 n_{s} \Delta n(x, y)$. In this case, the inverse algorithm for calculating the index profile is written in the form of ${ }^{3,8}$

$$
\Delta n(x, y) \approx-\frac{1}{2} \frac{E^{\prime \prime}}{n_{s} k^{2} E}+\Delta n_{\mathrm{eff}},
$$

where $k$ is the free space wave number and $\Delta n_{\text {eff }}$ is the difference between $n_{\text {eff }}$ and $n_{s}$. As only the intensity distribution is measured, Eq. (1) is rewritten as

$$
\begin{aligned}
\Delta n(x, y) \approx & -\frac{1}{2} \frac{(\sqrt{I})^{\prime \prime}}{n_{s} k^{2} \sqrt{I}}+\Delta n_{\mathrm{eff}}=-\frac{1}{4 n_{s} k^{2}}\left[\frac{I^{\prime \prime}}{I}-\frac{1}{2} \frac{\left(I^{\prime}\right)^{2}}{I^{2}}\right] \\
& +\Delta n_{\mathrm{eff} .}
\end{aligned}
$$

Hence, simultaneous measurements of $I, I^{\prime}$, and $I^{\prime \prime}$ are necessary for accurate reconstruction of the index profile. For a two-dimensional index profile, Eq. (2) can be written as

$$
\Delta n(x, y) \approx-\frac{1}{4 n_{s} k^{2}}\left[\frac{I_{x x}}{I}+\frac{I_{y y}}{I}-\frac{1}{2} \frac{\left(I_{x}^{2}+I_{y}^{2}\right)}{I^{2}}\right]+\Delta n_{\mathrm{eff}},
$$

where $I_{x}$ and $I_{y}$ are the first derivatives of $I$ in the $x$ and $y$ directions, respectively. $I_{x x}$ and $I_{y y}$ are the corresponding second derivatives of $I$.

The measurements of intensity profile and its derivatives are fulfilled by a DNSOM system, as shown in Fig. 1. The system was built on an atomic-force microscope modified with a tapered fiber probe. ${ }^{9}$ A $632.8 \mathrm{~nm}$ wavelength $\mathrm{He}-\mathrm{Ne}$ laser, modulated by an optical chopper at frequency $f_{0}$ $(=390 \mathrm{~Hz})$, was coupled into the optical waveguide. At the output end, the fiber probe was allowed to scan over the waveguide area within the near-field region by using a tuning-fork feedback mechanism. ${ }^{10}$ The probe is a tapered fiber coated with a gold layer of thickness $80 \mathrm{~nm}$ around the tip region to enhance the resolution. The tip has a diameter of $100 \mathrm{~nm}$, and therefore subwavelength resolution can be obtained in the near-field measurement. The probe was mounted on a quartz tuning fork, which oscillates the probe along the $x$ or $y$ direction with an amplitude at frequency $f_{1}$ 


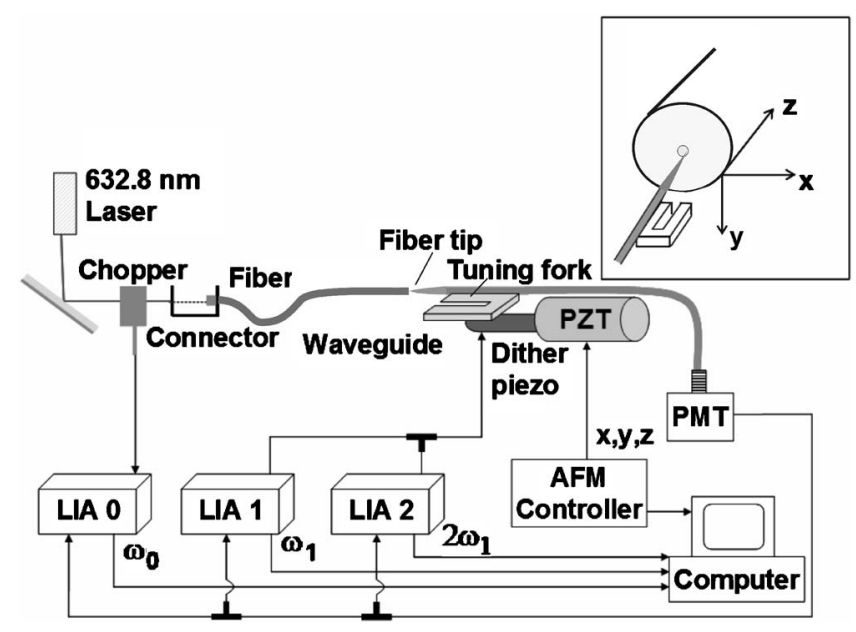

FIG. 1. System setup of the differential near-field scanning optical microscopy.

$(=33.48 \mathrm{kHz})$. The probe was connected to a photomultiplier tube (Hamamatsu, R928) and the signal was read by three lock-in amplifiers (LIAs), referenced at frequencies $f_{0}, f_{1}$, and $2 f_{1}$, respectively. The amplitude and phase signals were then sent to the computer to render the near-field images.

If the probe is vibrated in the $x$ direction at $\omega_{1}\left(=2 \pi f_{1}\right)$, the vibration is described by $x(t)=x(0)+\Delta x \sin \omega_{1} t$. The optical intensity $I(x)$ on the detector is approximated by

$$
\begin{aligned}
I[x(t)] \approx & I[x(0)]+\Delta x I_{x}[x(0)] \sin \omega_{1} t \\
& +\frac{1}{4} \Delta x^{2} I_{x x}[x(0)] \cos 2 \omega_{1} t .
\end{aligned}
$$

Hence, during the scan of the fiber tip, the image signal from LIA0 gives the optical intensity $I$ as that of the conventional

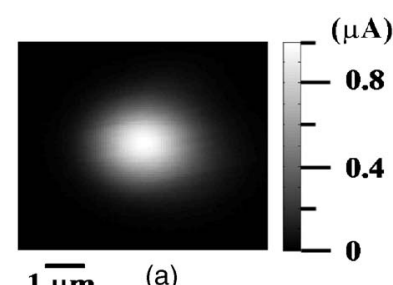

$1 \overline{\mu \mathbf{m}} \quad(\mathrm{a})$

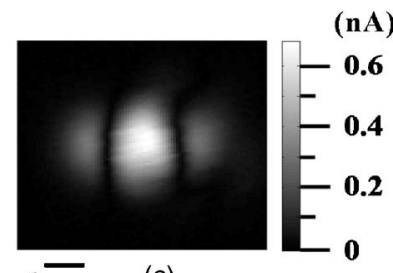

$\mathbf{1} \overline{\mu \mathbf{m}} \quad$ (c)

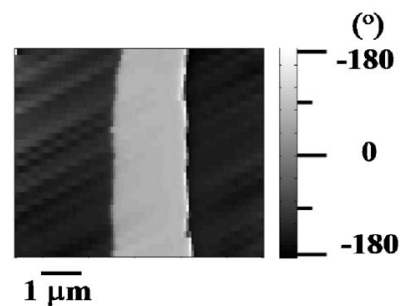

(e)

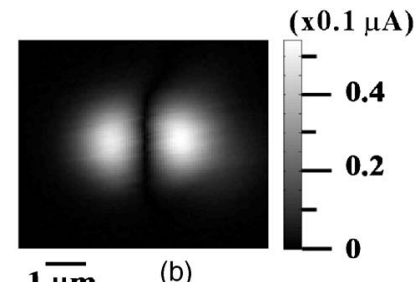

$\mathbf{1} \overline{\mu \mathbf{m}}$

(b)

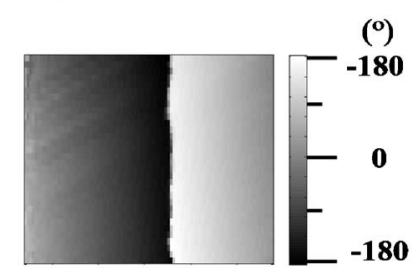

$\mathbf{1} \overline{\boldsymbol{\mu m}} \quad(\mathrm{d})$

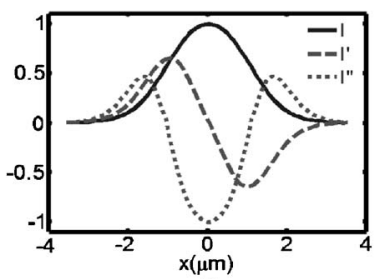

(f)
FIG. 2. (a) Measured image of output field intensity. (b) Measured image of the first derivative of output field intensity. (c) Measured image of the second derivative of output field intensity. (d) The phase image of (b). (e) The phase image of (c). (f) Measured intensity profiles, $I, I_{x}$, and $I_{\mathrm{xx}}$ measured field. (iv) Our DNSOM method. Downloaded 16 Feb 2009 to 140.112 .113 .225 . Redistribution subject to AIP license or copyright; see http://apl.aip.org/apl/copyright.jsp

method, whereas the image signals from LIA1 (referenced at $\omega_{1}$ ) and LIA2 (referenced at $2 \omega_{1}$ ) generate optical intensity derivatives $I_{x}$ and $I_{x x}$, respectively, as required by the proposed method. Similarly, the $I_{y}$ and $I_{y y}$ are obtained by vibrating the probe at $y$ direction. For $x-y$ symmetrical structure, $I_{y}$ and $I_{y y}$ can be obtained directly by rotating the images of $I_{x}$ and $I_{x x}$ for simplicity.

To demonstrate the application of two-dimensional refractive index reconstruction, a single mode fiber (3M, FS-SN-3224) with a core diameter of $3.2 \mu \mathrm{m}$ and a step index profile of $\Delta n$ equals to 0.005 at the laser wavelength of $632.8 \mathrm{~nm}$ is measured. Figures 2(a)-2(c) show the measured amplitude images of $I, I_{x}$, and $I_{x x}$, respectively. Figures 2(d) and 2(e) are the phase images of $I_{x}$ and $I_{x x}$ in Figs. 2(b) and 2(c). The distributions of the intensity derivatives are obtained by multiplying the measured amplitude derivatives with their phase signals. Moreover, according to Eq. (5), the measured first derivative of the intensity is equal to $I_{x} \Delta x$ and the measured second derivative is equal to $I_{x x} \Delta x^{2} / 4$, where $\Delta x$ has to be determined. We measured the $\Delta x$ by a small amplitude measurement method. ${ }^{11}$ It is notable that magnitude of $\Delta x$ is important. If it is too small, the signal-to-noise ratio will be too low. If it is too large, Eq. (4) will be inaccurate. In principle, $I_{x x}$ is proportional to $I(\Delta x / d)^{2}$, where $d$ is the width of the optical spot. In our system, the photomul-

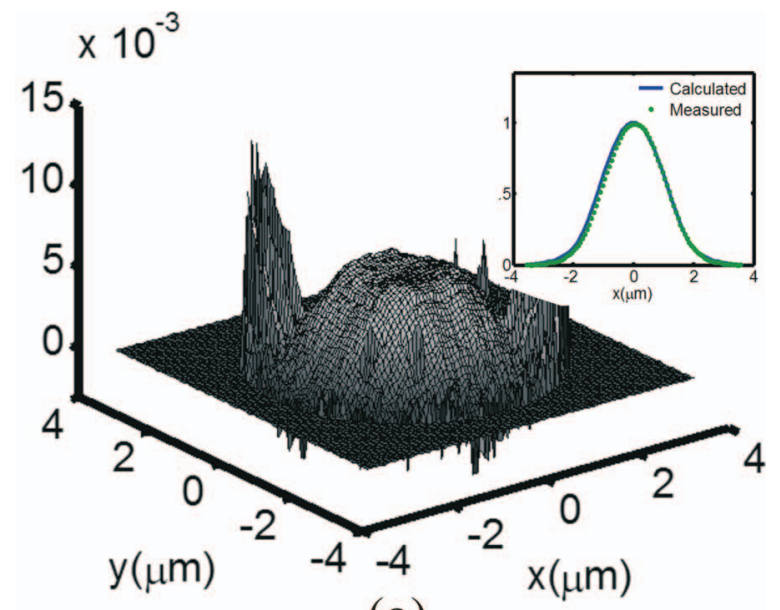

(a)

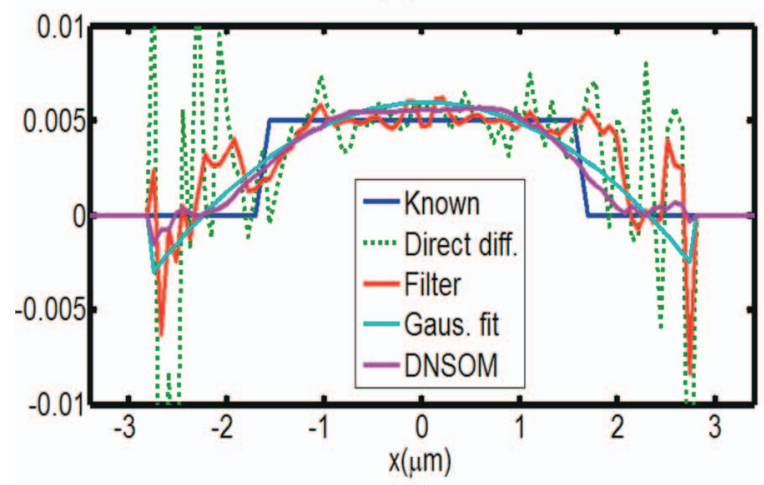

(b)

FIG. 3. (Color) (a) Reconstructed index profile of the single mode fiber. The inset shows a comparison of the optical field calculated by the known index profile and the measured optical field. (b) The cross section of the index profiles reconstructed from different methods. (i) Direction differentiations of the measured intensity profile. (ii) The intensity profile was smoothed by a Butterworth low-pass filter of order 3. (iii) Fitting a Gaussian profile to the
measured field. (iv) Our DNSOM method. 
tiplier tube has an input noise of $9.7 \times 10^{-16} \mathrm{~W} / \sqrt{\mathrm{Hz}}$ and the optical power in the fiber probe is $10 \mathrm{nW}$. In order to have a signal-to-noise ratio larger than 100 at $33.48 \mathrm{kHz}$, the $\Delta x / d$ should be larger than 0.04 . The optical spot of a single mode fiber is about $3 \mu \mathrm{m}$. We thus control the driving voltage to set $\Delta x$ to be $0.12 \mu \mathrm{m}$. By considering the phase signals and vibration amplitude, the actual distributions of the intensity derivatives are obtained. A cross-sectional plot of $I, I_{x}$, and $I_{x x}$ rendered from measured images are shown in Fig. 2(f).

Using Eq. (3), the reconstructed index profile of the single mode fiber is shown in Fig. 3(a). In this figure, the index profile near the core has a uniform distribution, consistent with the index distribution of the core of a fiber. It is noted that we do not apply any fitting function and smoothing technique in the index reconstruction. The measured intensity and its derivatives are close to zero in the cladding region. The zero/zero operations in Eq. (3) result in large noises in this region. However, these noises do not affect the accuracy of the main waveguide region. The inset in Fig. 3(a) shows the intensity distribution of the optical fiber, calculated by using the known index profile, and the measured intensity profile. Both intensity profiles are quite well matched. However, even with such matched intensity profile, the index profile cannot be well reconstructed without measuring its first and second derivatives. For example, Fig. 3(b) shows the cross section of the index profiles reconstructed from different approaches. The index profile calculated by directly differentiating the intensity profile has very large noises as expected. A low-pass filter can reduce the index fluctuations. However, it suffers from large errors at the core/ cladding interface, because high frequency components are lost. The fitting of a Gaussian function to the measured profile makes the smoothest distribution, but the Gaussian index profile shows a deviation to the step index distribution. Compared to above approaches, our method does not need any modifications on the measured field. It has the best fitting to the known index profile. The index fluctuation in the waveguide area is very small. The size of the profile and the magnitude of index are in good agreement.

In summary, a method for simultaneously measuring the eigenmode distribution of an optical waveguide and its first and second derivatives by a differential near-field scanning optical microscopy system is proposed. The proposed method not only avoids numerical differentiations in the inverse algorithm but also provides a subwavelength resolution. Therefore, a more accurate refractive index profile can be obtained, as compared with that by the conventional method. This makes the inverse calculation method simpler and feasible for comparisons of different shapes of the refractive index profiles. A single mode fiber with a step index for the laser wavelength of $632.8 \mathrm{~nm}$ is measured. Experimental results show that the reconstructed index profile is in good agreement with the known one.

This work was supported by National Science Council, Taipei, Taiwan, under Contract No. NSC-95-2215-E-002012 .

${ }^{1}$ I. Mansour and F. Caccavale, J. Lightwave Technol. 14, 423 (1996).

${ }^{2}$ F. Caccavale, P. Chakraborty, A. Quaranta, I. Mansour, G. Gianello, S. Bosso, R. Corsini, and G. Mussi, J. Appl. Phys. 78, 5345 (1995).

${ }^{3}$ D. Brooks and S. Ruschin, IEEE Photonics Technol. Lett. 8, 254 (1996).

${ }^{4}$ F. Caccavale, F. Segato, I. Mansour, and M. Gianesin, J. Lightwave Technol. 16, 1348 (1998).

${ }^{5}$ D. P. Tsai, C. W. Yang, S. Z. Lo, and H. E. Jackson, Appl. Phys. Lett. 75, 1039 (1999).

${ }^{6}$ A. L. Campillo, J. W. P. Hsu, C. A. White, and C. D. W. Jones, Appl. Phys. Lett. 80, 2239 (2002).

${ }^{7}$ J. Shakya, J. Y. Lin, and H. X. Jiang, Appl. Phys. Lett. 84, 1832 (2004).

${ }^{8}$ J. Helms, J. Schmidtchen, B. Schüppert, and K. Petermann, J. Lightwave Technol. 8, 625 (1990).

${ }^{9}$ R. Stöckle, C. Fokas, V. Deckert, R. Zenobi, B. Sick, B. Hecht, and U. P. Wild, Appl. Phys. Lett. 75, 160 (1999).

${ }^{10}$ K. Karrai and R. D. Grober, Appl. Phys. Lett. 66, 1842 (1995).

${ }^{11}$ C. C. Wei, P. K. Wei, and W. Fann, Appl. Phys. Lett. 67, 3835 (1995). 